Наведені методи контролю якості вугілля. Для підпалювання вугільного факела необхідні розпалювальні мазутні або газові форсунки. Ці форсунки можуть також включатись при погасанні основних пальників. Необхідний індивідуальний контроль за роботою кожного пальника у всьому діапазоні навантажень $і$ режимів агрегату. Завданням розробки є удосконалення пристрою контролю для подальшого автоматичного керування процесом спалювання вугілля

Ключові слова: факел, пальник, оптичний контроль, продукти горіння, пиловугільне паливо, спектр, топка

口- $\square$

Приведены методы контроля качества угля. Для поджигания угольного факела необходимы растопочные мазутные или газовые форсунки. Эти форсунки могут также включаться при погасании основных горелок. Необходим индивидуальньй контроль за работой каждой горелки во всем диапазоне нагрузок и режимов агрегата. Задачей разработки является усовершенствование системы контроля для дальнейшего автоматического управления процессом сжигания угля

Ключевые слова: факел, горел$\kappa a$, оптический контроль, продукты горения, пылеугольное топливо, спектр, топка

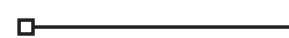

UDC 621.182 .26

DOI: $10.15587 / 1729-4061.2017 .119239$

\section{DEVELOPMENT OF AN IMPROVED DEVICE TO CONTROL FLAME BRIGHTNESS IN COMBUSTION CHAMBERS OF STEAM BOILERS}

\author{
O. Melnik \\ $\mathrm{PhD}$, Associate Professor* \\ E-mail: melnikolgaev@gmail.com \\ R. Park home n k o \\ Senior Lecturer* \\ E-mail: parchom@i.ua \\ O. S h choki n a \\ Senior Lecturer* \\ E-mail:_olina_@ukr.net \\ O. A n i s k ov \\ Assistant* \\ E-mail: aniskov@softproject.com.ua \\ Y. T s i bu l e v sk y \\ $\mathrm{PhD}$, Associate Professor \\ LLC "Rudpromgeofizika" \\ Kryvbasivska str., 54/12, Kryvyi Rih, Ukraine, 50000 \\ E-mail: tsibulevsky@ukr.net \\ O. Kharitonov \\ Lecturer \\ Ukrainian Polytechnic College \\ Karla Marksa ave., 66, Kryvyi Rih, Ukraine, 50000 \\ E-mail: Ckariton@i.ua \\ O. O melchenko \\ $\mathrm{PhD}$
}

Department of general engineering disciplinesan dequipment

Donetsk National University of

Economicsand Trade named after Michael Tugan-Baranowsky

Tramvayna str., 16, Kryvyi Rih, Ukraine, 50005

E-mail: omelchenko@donnuet.edu.ua

V. Chorna

$\mathrm{PhD}$

Department of systems of power consumption and energy management

Kremenchuk Mykhaylo Ostrohradskyi National University

Pershotravneva str., 20, Kremenchuk, Ukraine, 39600

E-mail: chornajav@gmail.com

*Department of power supply and energy management

Kryvyi Rih National University

Vitaliya Matusevycha str., 11, Kryvyi Rih, Ukraine, 50027

\section{Introduction}

In the Ukrainian energy sector, about 200 billion $\mathrm{kWh}$ of electricity is produced annually. At the same time, almost half of this energy is generated by thermal power plants and power stations.
In recent years, the heat from coal combustion has decreased significantly, its ash content has increased 2.5 times, and the sulfur content has increased substantially, too. All this reduces the possibility of using pulverized coal only and requires using additional fuels such as natural gas and black residual oil. Despite the fact that coal burning is much 
more profitable than burning natural gas, it is necessary to increase the quality of coal by reducing its ash content. To increase the combustion efficiency of coal, a suspension is produced from it and burnt in a high-temperature pulverized coal flame. The disadvantage of the process is the significant cost of coal enrichment and transportation.

In order to improve fuel quality, in 2012 the new State Standard DSTU 4083-2012 "Coal and Anthracite for Pulverized Burning at Thermal Power Plants" was adopted [1].

For automatic control of the coal composition, the flow is based on the methods of absorbing or dispersing electromagnetic radiation, ultrasound, charged particles, the natural radioactivity level of the accompanying rocks and neutron activation or powerful laser activation.

It is important to develop a device to control fire brightness as it provides the possibility of safe and economical control of the operating mode of the boiler furnace.

The firm that has been developing flame control sensors for more than 20 years emphasizes that the existing sensors [2]:

- have insufficient reliability of controlling the presence and absence of flames;

- have low selectivity in determining a fading burner;

- are sensitive to external illumination;

- cost much.

The flame indicator must have high reliability and selectivity for igniting or extinguishing the torch, as well as the absence of reaction to any other sources of fire. The great difficulty is the uneven burning of the flame along the torch. The dust burner is heated by means of a black fuel nozzle. The operation of the burner control should be carried out in accordance with the operation of the dust system: the entire group of burners, which is powered by one dust system, must be switched on or off simultaneously with the system. It is necessary for the signal value of the sensor to exceed the noise level significantly.

\section{Literature review and problem statement}

In recent years, optical pyrometers have been widely used, and their principle is based on registering a continuous spectrum of radiation with its maximum in the infrared region. In [3], to control temperature, an uncoated quartz light fiber is used in the combustion zone. Heated to the flame temperature, the input end of the optical information channel becomes a secondary source of radiation. In this case, the temperature range in the control zone is 1400-1863 degrees Celsius, and the wavelength of the radiation is $(3.0-4.5) \mu \mathrm{m}$. The disadvantage of this sensor is that the melting point of quartz is 1710 degrees, which leads to a fast failure of the sensor.

The analysis of the current level of combustion gauges shows that besides the spectral methods for controlling the temperature of flames and gases, an additional ionization or photocell is used to convert the electrical conductivity pulsation or brightness of the burner torch into an electrical signal that characterizes the radiation of the torch in the burner [4]. The degree of ionization and electrical conductivity of the gases of pulverized burners is influenced by the size of the burning particles and the content of impurities in the fuel. The disadvantage of an ionization sensor is the rapid failure of its electrodes. In this case, the factors that matter are the material from which the electrode is made, its shape, and its surface condition. The natural frequency of the flame flushing fluctuates around the average frequency of $3-4 \mathrm{~Hz}$. Fixation of the variable component of radiation allows reducing the effect of the constant heat field of the furnace walls on the accuracy of measurements [4]. To select the radiation spectrum of combustion products, optical filters are used, which are subdivided into strips that pass a narrow band of wavelengths of radiation, longwave and shortwave, and cut off the waves whose length is larger or smaller than the selected range. Light filters are chosen in such a way that they not only allocate the desired spectrum of radiation but also weaken the radiation of other combustion products [5]. A more detailed choice of optical interference filters is possible only after determining the radiation spectrum of the main combustion products. For the lens, it is better to choose conventional optical glass that has sufficient transparency in the visible and near-infrared spectrum, is well processed and has rather high physical and mechanical properties [5].

The method of selective control of the burner flame uses the ratio of the emission spectra of combustion products among themselves or in relation to the intensity of the radiation of the torch edges.

The installation of temperature sensors directly near the source of radiation is impossible, since their operating temperature does not exceed $125^{\circ} \mathrm{C}$. The large distance between the sensor and the radiation source filled with gases and combustion products becomes an additional source of measurement errors [4]. Therefore, the German company AMX ENGINEERING has equipped its sensors with heat-resistant light lines [6], which has brought the sensor's inlet to the source of radiation and has significantly increased the accuracy of measurements. However, the angle of view of these sensors is 6 degrees, which significantly reduces the control area of the torch [6]. The choice of light conductors, light filters and light detectors themselves depends on the emission spectrum of the combustion products. From the experience of leading companies, it is known that the control of combustion of gas and black fuel oil is mainly based on the ultraviolet spectrum of radiation; for coal, these factors are infrared radiation and part of the visible spectrum [6]. In [7, 8], optical sapphire fibers are used for light fibers, but the transparency region does not cover most of the spectrum.

The disadvantage of known devices is the low accuracy of control due to the lack of compensation for optical interference occurring in the space between the device and the object of control.

The basic scheme of a two-channel infrared temperature sensor is given in [9]. Its design, however, is not adapted for use in production conditions. It is known that the DURAG flame sensors of AMH ENGINEERING LLC have two logarithmic amplifiers [6]. This is due to the fact that in the circuit of the electronic unit to calculate the ratio of signals from two parts of the spectrum, it is necessary to obtain the difference between the logarithms of these two signals [10]. The disadvantage of this device is the lack of an automatic measurement correction input, taking into account the completeness of the combustion of coal fuels.

\section{The aim and objectives of the study}

The aim of the study is to determine the spectral and temperature characteristics of the products formed during the combustion of pulverized coal, which will increase the selectivity of the device to control the brightness of the flame. 
To achieve this aim, the following objectives are set to be done:

- to determine the emission spectrum of combustion products and temperature distribution along the torch;

- to develop a functional scheme of the device for controlling the brightness of the flame;

- to substantiate the principle of the device operation;

- to develop an electronic scheme of the flame brightness control device.

\section{Research and development of the device to control fire brightness in the furnaces of steam boilers}

\subsection{Determination of the emission spectrum of com-} bustion products and the temperature distribution along the torch

Depending on the intensity, the flame of the torch is divided into three temperature zones: initial, medium, and final. In [10], there is a band of the maximum radiation of combustion products in the infrared region of the spectrum: $\mathrm{H}_{2} \mathrm{O}-2.7 \mu \mathrm{m}, \mathrm{CO}_{2}-4.3 \mu \mathrm{m}, \mathrm{CO}-4.7 \mu \mathrm{m}$, nitrogen oxides in the amounts of $4.48 \mu \mathrm{m}, 2.87 \mu \mathrm{m}$, and $5.25 \mu \mathrm{m}$; sulfur oxides in the amounts of $7.28 \mu \mathrm{m}$ and $3.98 \mu \mathrm{m}$; and $\mathrm{CH}_{4}-3.32 \mu \mathrm{m}$. In addition, solid carbon particles (soot) have a continuous spectrum of radiation with a maximum of $0.9 \mu \mathrm{m}$, which depends on the temperature and size of the particles.

It is very interesting to explore the possibility of simultaneous control of $\mathrm{CO}$ and $\mathrm{CO}_{2}$ in relation to background bright temperatures [11]. However, the method is more suitable for use in the metallurgical industry.

In the initial part of the torch, fluctuations occur at a frequency of 800 to $1500 \mathrm{~Hz}$, and in the tail, the frequency is less than $200 \mathrm{~Hz}$. Spectra of individual components can be selected from the general spectrum of combustion products by using optical light interference filters and reducing the diaphragm opening [4]. With these filters, it is possible to select a band ranging from 1.0 to $10 \mathrm{~nm}$.

\subsection{The functional scheme of the device to control} fire brightness

The functional scheme of the flame brightness control device is shown in Fig. 1.

The device contains sequentially connected optical elements located in a diaphragm tube. The outputs of light detectors through electronic amplifiers and frequency spectrum analyzers are connected to the corresponding inputs of a logic block. The output of the logic block is connected to the output block (20). In this case, the electronic amplifiers, frequency spectrum analyzers, logic block and output block are located in a remote electronic unit.

\section{3. The principle of the flame brightness control device}

The diaphragm tube (1) protects the optical system from extraneous light and pollution. The lens (2) generates a torch image in the input plane of the optic fiber distributor (3) that divides it into three channels. The condensers $(4,5$, and 6 ) are intended for radiation concentration at the light detectors $(10,11$, and 12). With the help of the interference filters $(7,8$, and 9$)$, the spectral distribution of the torch radiation is performed according to the corresponding optical channels. The light detectors $(10,11$, and 12) convert the radiation energy of the torch into an electrical signal amplified by the electronic amplifiers (13, 14, and 15). The spectrum analyzers of the frequencies $(16,17$, and 18) filter the pulsation frequencies characteristic of the individual parts of the torch flame. The logic block (19) is designed to generate monitoring and control signals as well as to transmit these signals through the output unit (20) to the control system.

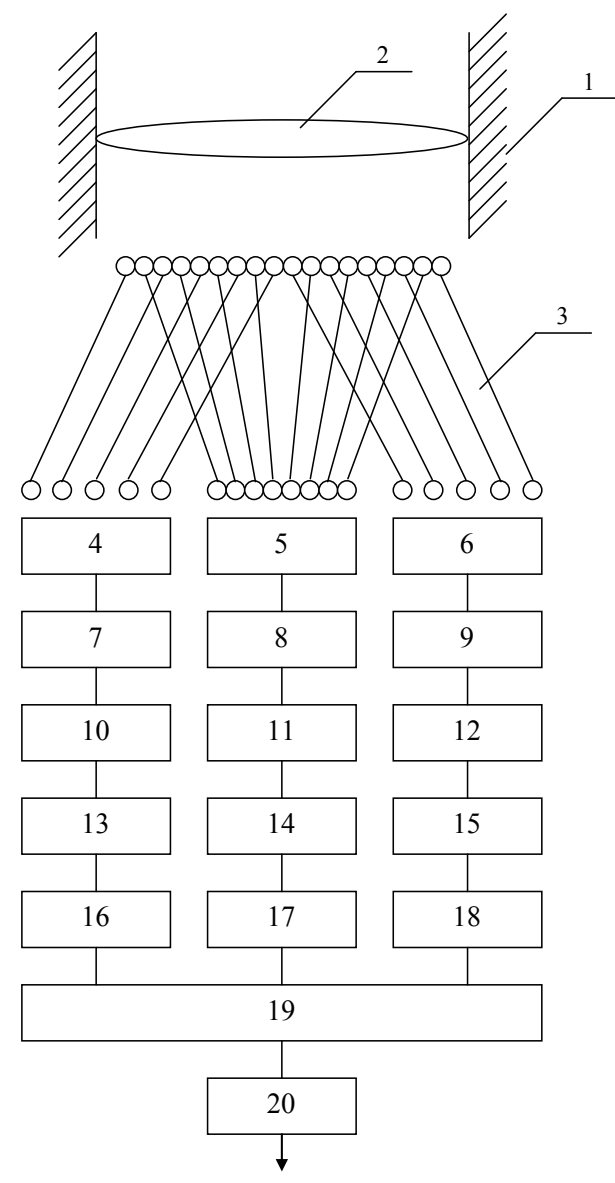

Fig. 1. The functional scheme of the device to control fire brightness: 1 - a tube; 2 - a lens; 3 - an optical distributor, made in the form of an optical fiber transformer;

4, 5, and 6 - condensers; 7, 8, and 9 - interference filters; 10,11 , and 12 - light detectors; 13,14 , and 15 - electronic amplifiers; 16,17 , and 18 - spectrum analyzers; 19 - a logic block; 20 - an output block

In the zone of the torch, products with a band of maximum radiation in the infrared spectrum range from 2.82 to $5.27 \mu \mathrm{m}$ are formed. When performing selective control, it is possible to use the above lines for one of the products of combustion with the use of light detectors 10 and 12 . For the light detectors, the optical fiber divider splits the area of the torch image in two. The ratio of the output signals of these two channels helps make conclusions about the brightness of the flame. To control the concentration of solid particles (soot, ash), which are formed in the space between the device and the object of control, an additional channel has been introduced. In the channel to light detector 11, through the optic fiber distributor (3) and through condenser 5 and interference filter 8 , there appears a full image of the torch flame. In this case, interference filter 8 and light detector 11 are estimated for a wavelength equal to $0.9 \mu \mathrm{m}$.

The spectral absorption coefficient for such a medium can be determined analytically. When the optical size of the particles is changed from 5 to $30 \mu \mathrm{m}$, the spectral absorption 
coefficient recursively changes in this range. With an unlimited increase in the size of the particles and a reduction in the wavelength of the incident radiation, the particles in their optical properties are similar to large opaque screens. The effect of the concentration of solids on the degree of reducing the transparency of the medium is taken into account by the logic block (19) in diagnosing the actual flame brightness and applying a control signal to the output block (20). If the signal ratio decreases to $10 \%$, the output unit (20) indicates the flame fading.

For more precise control of the combustion mode, an additional selection of signals is used with spectrum analyzers of the flux pulsation $(16,17$, and 18).

The introduction of an additional channel to control the value of interference can help make an adjustment for the effect of changing the optical properties of combustion products on the results of measurements, which increases the accuracy of control.

The performance of the proposed device depends on the correct optical material. The optimal spectral signal ratio begins to be provided with the choice of filters. The greatest accuracy can be obtained with the help of interference filters that transmit wide bundles of light up to 10-20 degree angles. The maximum filter passage corresponds to the wavelengths for which the thickness of the dielectric layer is multiplied by half the wavelength.

The disadvantage of the existing system is the large gap between the PECs (photoelectronic converters) and their location near the torch burner. These shortcomings can be prevented by means of a special optic fiber transformer that provides high transmittance and decomposes the image into elements, which makes it possible to implement any encryption law and enlarge the image. The value of the transmission coefficient of the optical fiber wiring binder is directly proportional to the total useful area of the ends of the fibers [5]:

$$
\eta=\frac{S_{1}}{S_{0}} \tau\left(1-\rho^{2}\right) \sin U
$$

where $s_{1}$ is the total useful area of the ends of the fibers; $s_{0}$ is total area of the input end of the binder, including the intervals between the fibers; $\tau$ is the coefficient of fiber conduction; $\rho$ is the coefficient of reflection on the ends of the fiber; $U$ is the aperture angle at the entrance.

Depending on the temperature range, light detectors and optical fibers are selected. The advantages of an optical fiber as a structural element in the connection system are the following: the broadband up to one terohertz; small losses down to $0.154 \mathrm{~dB} / \mathrm{km}$; a fiber diameter of $125 \mu \mathrm{m}$; absence of cross-noise and high corrosion resistance. The transparency range of the optical fiber from quartz glass is $0.4 \ldots 2.0 \mu \mathrm{m}$, and the temperature range is up to 2000 degrees Celsius.

The use of a sapphire optical fiber in coal burners is not justified because the emission spectra of combustion products do not fully coincide with its "window" of transparency [11]. The maximum transparency is the highest with a wavelength of $2.94 \mu \mathrm{m}$. A multicomponent glass fiber is made of ordinary glass with impurities of germanium oxides, lithium, and magnesium. Losses are $4 \mathrm{~dB} / \mathrm{km}$ at a wavelength of $0.8 \mu \mathrm{m}$; the melting temperature is 1400 degrees Celsius. A plastic fiber is optimal for use in systems where a light diode is the source of light. An infrared fiber is an optical fiber with low losses in the region of infrared waves of the length from 2 to $10 \mu \mathrm{m}$. For the construction of an optical fiber transformer, we choose an optical fiber made of quartz glass, the transparency of which is maximal in the near-infrared radiation. An optical fiber can be used to bring an image to a television camera from several torches at the same time; the method is especially effective for color television. A promising direction is the combined use of optical fibers with mosaic light detectors.

The choice of the radiation detector is based on spectral relations, which depend on the radiation wavelengths of the individual products of the torch flame and the intensity of the background radiation, isolated by means of light interference filters. The magnitude of the electrical signals that correspond to the light flux from the flare section and the area of the background barrier is calculated by the formulae [5]

$$
\begin{aligned}
& u_{1}=k_{1} \int_{\lambda_{1}}^{\lambda_{2}} s_{\lambda}^{\prime} \tau_{\lambda} r_{\lambda u}^{\prime} \mathrm{d} \lambda . \\
& u_{2}=k_{2} \int_{\lambda_{1}}^{\lambda_{2}} s_{\lambda}^{\prime} \tau_{\lambda} r_{\lambda \phi}^{\prime} \mathrm{d} \lambda,
\end{aligned}
$$

where $\tau_{\lambda}$ is the transmission coefficient of the medium; $r_{\lambda}$ is the radiation density; $s_{\lambda}$ is the spectral sensitivity: $s_{\lambda}^{\prime}=s_{\lambda} / s_{\lambda \max }$ and $r_{\lambda} / r_{\lambda_{\max }}$.

The constants $k_{1}$ and $k_{2}$ do not depend on the radiation wavelength; they mainly include the characteristics of the emitters and the optical system $\left(s_{\text {rad }}, l_{\text {rad }}, s_{\mathrm{t}}, l_{\mathrm{t}}, s_{\mathrm{in}}, r_{\lambda \max }\right)$, as well as some parameters of the radiative energy receiver, for example its maximum spectral sensitivity $s_{\lambda_{\max }}$.

The values of the integrals in expressions (2) and (3) can be easily found using the simplest graph analytics methods. To do this, it is necessary to construct the dependences $\tau_{\lambda}$, $r_{\lambda u}^{\prime}$ and $s_{\lambda}^{\prime}$. on the same scale and in relative units. Then it is necessary to construct the curves $s_{\lambda}^{\prime}, \tau_{\lambda}, r_{\lambda u}^{\prime}$ and $s_{\lambda}^{\prime}, \tau_{\lambda}, r_{\lambda \phi}^{\prime}$ and to find their areas $Q_{u 1}$ and $Q_{u 2}$ in the pre-selected range $\lambda_{1}-\lambda_{2}$. If for the same graphs there is a construction for several receivers, then by comparing the ratio of the areas $Q_{u 1}$ and $Q_{u 2}$, it is possible to choose an optimal one among them in terms of spectral relations in the range $\lambda_{1}-\lambda_{2}$.

By selecting the range $\lambda_{1}-\lambda_{2}$ in advance, it is possible, without having to choose the receiver, to find the spectral characteristics of the transmission of the optimal filter. Taking into account the spectral characteristics, it is further possible to select the receiver according to the above methodology. In this case, it becomes immediately possible to calculate the absolute values of signals from the observed objects, from obstacles and backgrounds, that is, to find the value of the ratio $m=$ signal/obstacle.

In modern devices for controlling the combustion (dying out) of the flame, detectors are represented by photoresistors and photodiodes with small dimensions, high sensitivity, and reduced power supply [5]. The disadvantage of photoconductors is increased inertia, dependence of their parameters on temperature, and insufficient linearity of light characteristics. Photodiodes are made of germanium and silicon. Silicon diodes are more stable in germanium at changing temperature, humidity, and pressure. The integral sensitivity of silicon diodes reaches $7 \mathrm{~mA} / \mathrm{lm}$, which is three times greater than the germanium sensitivity. To control the combustion process, it is better to use avalanche silicon diodes in a photovoltaic mode or mosaic matrices. It is better to adjust the sensors to the variable component of radiation, which helps eliminate the effect of constant radiation from the heated surfaces of the walls. 
The protective tube of the device is introduced into the combustion chamber through the wall of the furnace and is installed at an angle close to 15 degrees facing the torch. Increasing UV radiation indicates an increase in temperature, and an increase in the infrared component means incomplete combustion of fuel. In low-power furnaces, the choice of the integral radiation of the furnace walls as a reference signal is not reliable because the total intensity is very much dependent on the mode of each individual burner. In large furnaces, the torches flutter well to differ from the walls of the furnace, the radiation of which practically has no fluctuation. The spectral sensitivity of the main photodetectors is in the region of $2.0-3.0 \mu \mathrm{m}$. For increased sensitivity, the photodiodes can be equipped with an electronic thermostat to maintain a temperature of about 20 degrees Celsius with an accuracy of 0.5 degrees.

\section{4. An electronic block diagram}

Study [9] provides a scheme of temperature control by the spectral optical method. The disadvantage of the proposed scheme is the absence of automatic measurement correction depending on the concentration of secondary combustion products.

Article [12] proposes to use artificial vibrations of the burner radiation and registration in the spectrum of $470-630 \mathrm{~nm}$. The images are digitized and compared in time, and area changes determine the degree of the flame propagation. This solution complicates the design and reduces the speed of the device. For the processing of optical information, a remote electronic unit (Fig. 2, a) is developed to consist of light detectors, amplifiers, as well as logic and output blocks. Optical fibers are collected in a drum and divided into three groups, which, by means of interference filters, emit radiation with wavelengths $\lambda_{1}, \lambda_{2}$, and $\lambda_{3}$. These components of radiation come to the converters of light streams of photodiodes PD1 and PD2 as well as phototransistor PT. In order to determine the ratio of the two components of the combustion products, the flowchart is supplied with two logarithmic amplifiers of thermal compensation with the help of additional transistors V2 and V4.

The flowchart of the electronic unit of the flame brightness control device and the output characteristics of the logic block are shown in Fig. 2.

These components of infrared radiation are applied to light signal detectors - photodiodes and phototransistors, which in Fig. 2 are marked as PD1, PD2, and V1. In order to determine the ratio of the radiation of the two components of combustion products, two logarithmic amplifiers are introduced in the circuit on the basis of Op-Amp1 and Op-Amp3 with thermal compensation with the help of additional transistors V2 and V4. The output voltage for this circuit is determined by the formula

$$
U_{\text {out }}=-\varphi \ln I_{\mathrm{PD}} / I_{\text {com } 1},
$$

where $\mathrm{I}_{\text {com } 1}$ is a compensation circuit that can be set using a resistor R1 [10].
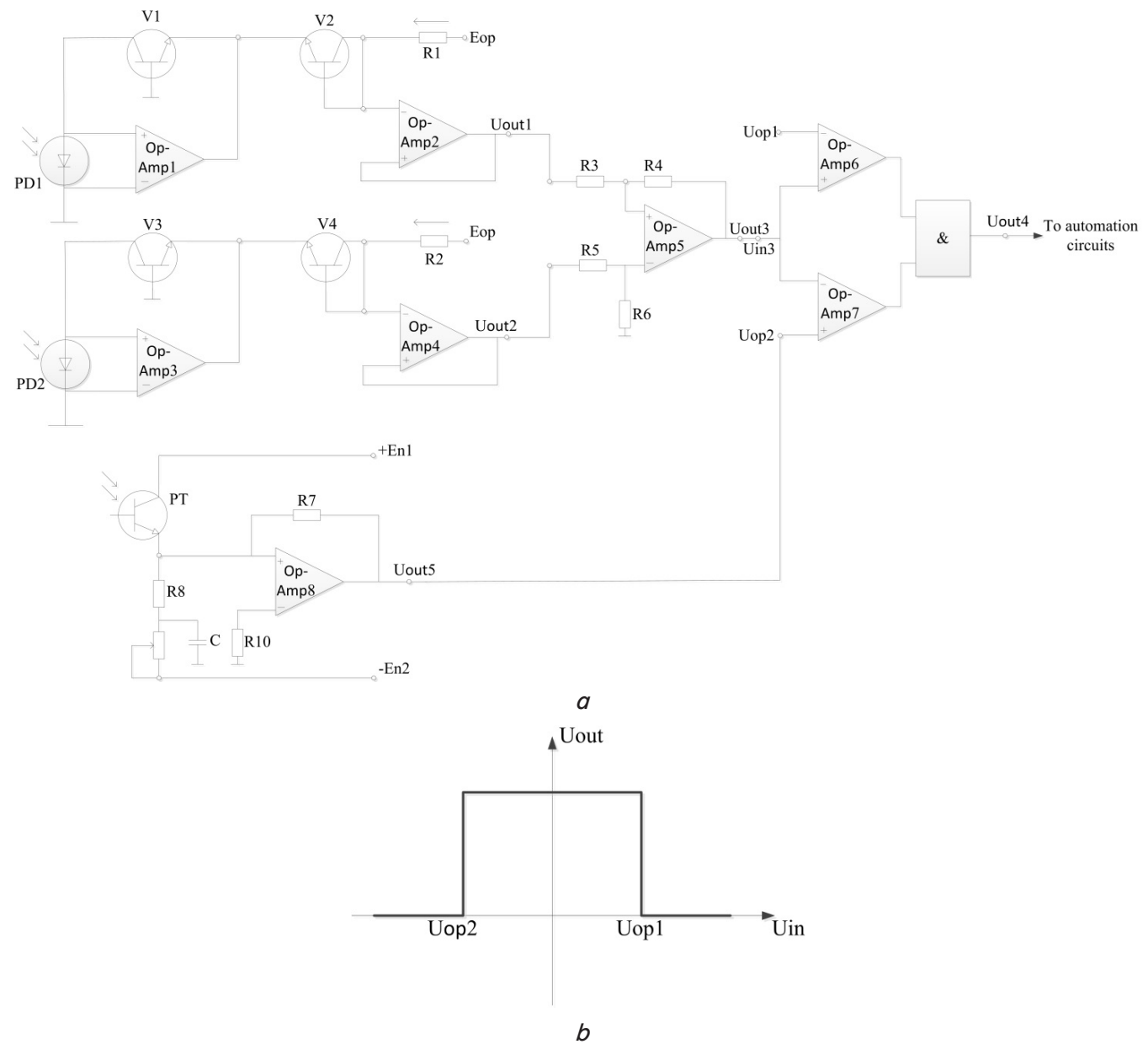

Fig. 2. The device to control fire brightness: $a-$ the circuit of the electronic unit; $b-$ the initial characteristics of the logic block 
The ratio of the input voltage from the light detectors PD1 and PD2 is determined by the formula

$$
\frac{U_{\mathrm{PD} 1}}{U_{\mathrm{PD} 2}}=\ln \left(U_{\text {out }}-U_{\text {out } 2}\right)
$$

The algorithm of calculation can be done using a differential amplifier based on Op-Amp5. Its output voltage is regulated using $\mathrm{R} 3$ to correct the effect of $\mathrm{CO}_{2}$ on the accuracy of the intensity measurements of the ratio $\lambda_{1} / \lambda_{2}$ of the photodiodes PD1 and PD2.

The output voltage is determined by the formula

$$
U_{\text {out }}=\frac{R 2}{R 1}\left(U_{\mathrm{in} 2}-U_{\mathrm{in} 1}\right)
$$

where $R 1 \cdot R 2=R 2 \cdot R 3$ [5].

The logic output block is introduced to isolate the insensitivity zone, which has to be provided with hardware. Another introduction is the input voltage device of the "less, norm, more" type based on operational amplifiers Op-Amp6 and Op-Amp7. By changing the levels of the reference signals $U_{\text {op-amp1 }}$ and $U_{\text {op-amp2 }}$, it is possible to form the required width of the "window" on the original characteristics of the device (Fig. 2,b) [10]. The reference voltage Uop-amp1 can be considered constant, and the width of the "window" is measured from it. The photoamplifier Op-Amp8 with a phototransistor PT measures the radiation intensity of interference products with a wavelength $\lambda_{3}$.

The output voltage of the photoamplifier Op-Amp8 is determined by the formula:

$$
U_{\text {out }}=-R 1 I_{R 1} \text {, }
$$

where $I_{R 1}$ is the current flowing through R1 [10].

The output voltage of the photoamplifier Op-Amp8 is regulated by resistor $\mathrm{R} 3$ and used to specify the reference voltage $U_{\text {op-amp2 }}$ at the input of Op-Amp6 to correct the impact of interfering combustion products on the accuracy of determining the relationship between the radiation intensity of the main combustion products. The output of the logic element " $I$ " is connected to the circuits of automatic control of the combustion process.

\section{Discussion of the research results of the design of the proposed device}

The use of high-temperature glass fibers for transmitting an optical image of a torch makes it possible to bring the photodetector closer to the control point, where the temperature is more than 1500 degrees. When approaching the optical input of the sensor to the torch, the effect of combustion products and the neighboring burners on the accuracy of the control is reduced. In [3], it is suggested to heat the input end of the fiber sensor to the flame temperature and introduce it as a secondary source of radiation. Sensors D-LE 703 are produced by DURAG GROUP (Germany) with a long flexible optical fiber whose viewing angle is 6 degrees [6]. Both variants provide control of the flame on a small surface of the torch. A well-adjusted torch has a length of $0.8 \mathrm{~m}$ and is subject to turbulent effects all the time. The advantage of using an optical fiber transformer developed in this study is that it provides control of a much larger surface of the flame than the previous designs, which increases the accuracy of the control.

Known optoelectronic sensors usually have two optical channels that control the brightness of the radiation of two different combustion products and then, in relation to these measurements, determine whether the burner is burning or dying out. The proposed device is equipped with a third measuring channel for the constant compensation of radiation products that affect the accuracy of the control. The logic output block provides a zone of insensitivity, which allows selecting a "window" in the initial characteristics of the device to configure the device in furnaces with different burner locations and different operating temperatures.

The advantages of this design are the following:

- the use of optical fiber has brought the sensor inlet to the control zone, which has reduced the effect of the layer of smoke and water vaporization;

- the optical fiber transformer allows simultaneous control of the large surface of different parts of the torch;

- the design of the device and the electronic circuitry allow for the automatic correction of the radiation exposure of the combustion products that interfere with the control of the radiation of the main compounds.

The disadvantages of this work are the lack of research of the device in the real burners of steam boilers with different variants of the location of the burners, as well as the lack of a new trace element in the electronic circuit of the device.

The design of the fiber optic transformer and the main blocks of the electronic unit can be used to develop a series of combustion control devices for coal burners. In addition, the device can be used to control the process of agglomerate sintering [13].

This work is a continuation of the development of gamma-methods for controlling the ash content of coal on the conveyor, as well as the sensors of the total combustion of coal at Zelenodolsk TPP (Ukraine). There is a need to complete the proposed device constructively.

In further research, it is possible to use the sensitivity of the optical fiber directly to the temperature and to abandon the use of photodiodes and semiconductor matrices as infrared radiation detectors. To determine the temperature value of the sensors in the form of an optical fiber, the devices used are, in particular, Mach-Zender and Fabri-Perot interferometers. The basis of these methods is the thermal deformation of the optical fiber, as well as the fluctuation and intensity of the interference of light.

The selectivity of the device may not be sufficient when the burner is arranged in the opposite position. In this geometry, each burner must have its own control device the output of which will be connected to the output of the opposite device through a comparison circuit. Such a scheme will uniquely identify any fading burner.

The adaptation of the device in case when the coal torch goes out and the combined burner switches to combustion of gas or black fuel oil has not been considered. To do this, it will be necessary to calibrate the device on the radiation spectrum of another fuel and to provide for the automatic transfer of the device to a new measurement mode.

\section{Conclusions}

1. The analysis of the infrared radiation spectra of combustion products has shown that the accuracy of the control 
is influenced by the amount and size of carbon particles, which depends on the temperature of combustion.

2. The study has developed a functional scheme of the device to control fire brightness. The main element of the device is the design of an optical fiber transformer that provides simultaneous control of individual sections of the flame and the entire surface as a whole.

3 . The findings have substantiated the principle of the device operation based on the automatic introduction of the correction for the concentration of products that hinder the accurate calculation of the ratio of the main products of combustion.

4. An electronic block scheme has been developed to control the burner's operation according to the ratio of combustion product spectra, and the logic output block has a "window" for adjusting the device the characteristics of which depend on the furnace temperature and the location of the burners.

\section{References}

1. DSTU 4083-2012. Coal and anthracite for pyrolytic combustion at thermal power plants [Text]. - State Standard of Ukraine, 2012.

2. Flame sensors are one of the most important factors for the safe operation of the boiler house [Text] // Heat Supply News. - 2016. Issue 12 (164). - P. 125-132.

3. Shekhurdin, A. Use of optoelectronic systems with quartz monolithic light guides in industrial process control systems of industrial furnaces [Text] / A. Shekhurdin // Automation in the industry. - 2007. - Issue 4. - P. 23-24.

4. Pavlysh, O. Flame sensor for flame control. Tests on pulverized coal boiler TP-100 of unit 9 of Burshtyn TPP [Text] / O. Pavlysh // Energy and Fuel and Energy Complex. - 2008. - Issue 1.

5. Yakushenkov, Y. Fundamentals of the theory and calculation of optoelectronic devices [Text] / Y. Yakushenkov. - Moscow: Sovetskoe Radio, 1971. - 336 p.

6. Flame sensors DURAG [Electronic resource]. - Available at: http://www.amx-engineering.com/upl_files/catalog2/Datchiki_ plameni_Durag.pdf

7. Saari, R. A passive infrared sensor for combustion efficiency and process control [Text] / R. Saari. - M. A. Sc. University of Toronto, 2007. $-12 \mathrm{p}$.

8. Chen, K. P. Development of Metal Oxide Nanostructure-based Optical Sensors for Fossil Fuel Derived Gases Measurement at High Temperature [Text] / K. P. Chen. - Univ. of Pittsburgh, PA., 2015. - 49 p. doi: 10.2172/1172616

9. Infrared IR Sensor Circuit Diagram and Working Principle [Electronic resource]. - Available at: https://ru.scribd.com/ document/326171981/Infrared-IR-Sensor-Circuit-Diagram-and-Working-Principle

10. Shcherbakov, V. I. Electronic circuits on operational amplifiers [Text]: handbook / V. I. Shcherbakov, G. I. Grezdov. - Kyiv: Technique, 1983. - $213 \mathrm{p}$.

11. Coggin, J. New Optimal Sensor Suite for Ultrahigh Temperature Fossil Fuel Applications [Text] / J. Coggin, J. Ivasauskas, R. G. May, M. B. Miller, R. Wilson. - Prime Research Lc, 2006. - 35 p. doi: 10.2172/901547

12. Borzov, S. Selective control presence of a flame in boilers with the opposite location of the burners devices [Text] / S. Borzov, V. Kozik, Zh. Sheishenov // Heat power engineering. - 2009. - Issue 3. - P. 71-74.

13. Shchokin, V. Neuro-fuzzy activation sub-system of effective control channels in adaptive control system of agglomerative process [Text] / V. Shchokin, O. Shchokina // Metallurgical and Mining Industry. - 2015. - Issue 3. - P. 6-14. 